\title{
TAVI-in-TAVI in a patient after alcohol septal ablation
}

\author{
Eva Polaková, Radka Adlová, Petr Hájek, Josef Veselka \\ Charles University, Motol University Hospital, Prague, Czech Republic
}

\author{
Adv Interv Cardiol 2021; 17, 4 (66): 410-411 \\ DOI: https://doi.org/10.5114/aic.2021.112525
}

\section{Introduction}

Cardiovascular medicine at its beginning was dominated by surgical treatment options. The trend of the last decades is heading towards catheterization procedures. Alcohol septal ablation (ASA) was published by Sigwart in 1995 [1] whereas transcatheter aortic valve implantation (TAVI) was performed in 2002 for the first time [2]. Both methods have become efficient therapeutic options not only for patients not suitable for surgery but also for lowrisk patients [3-5]. Although the procedures are widely performed, certain challenges remain $[6,7]$. Moreover, as patients get older, new difficulties emerge.

We present a case of a patient with hypertrophic obstructive cardiomyopathy (HOCM) that was treated by ASA. Fifteen years later the patient developed severe aortic stenosis and was treated by TAVI that became severely stenotic 5 years later and required TAVI-in-TAVI implantation.

\section{Case series}

A 72-year-old woman presented to our outpatient clinic with shortness of breath as a main manifestation, heart failure New York Heart Association (NYHA) functional class III and nocturnal dyspnea. On the physical examination, the patient had rales heard over both lung bases, blood pressure was 130/70 $\mathrm{mm} \mathrm{Hg}$, heart rate was 70 beats/min with atrioventricular dual paced rhythm. The patient has an apple shape body habitus and short stature.

The patient has a history of HOCM, had a pacemaker implantation 30 years earlier and underwent ASA for left ventricular outflow tract obstruction 8 years afterwards. Moreover, she had symptomatic severe aortic stenosis 5 years ago. Based on the patient's preference and high surgical risk TAVI was performed using a LOTUS $23 \mathrm{~mm}$ (Boston Scientific, Marlborough, USA). The patient was prescribed dual antiplatelet therapy with aspirin and clopidogrel for 6 months followed by aspirin alone. On top of that, paroxysmal atrial fibrillation was detected and treated with amiodarone and rivaroxaban in addition to the treatment of heart failure.

Transthoracic echocardiography revealed a left ventricle ejection fraction (LVEF) of 35\%, Lotus aortic valve gradients were $53 / 29 \mathrm{~mm} \mathrm{Hg}$, iAVA 0.55 , the motion of the right coronary cusp was restricted, there was no aortic regurgitation, moderate mitral regurgitation and high probability of pulmonary hypertension with PASP above $50 \mathrm{~mm} \mathrm{Hg}$. Computed tomography (CT) of the heart showed calcification of the Lotus aortic valve, but there was no thrombosis or vegetation of the aortic valve.

Due to comorbidities and high surgical risk, the heart team recommended a TAVI-in-TAVI procedure. Estimated EUROSCORE II and STS score were $8.4 \%$ and $8.8 \%$ respectively. TAVI-in-TAVI under general anesthesia with transfemoral access was performed. First, a 23-mm Portico valve [8] was inserted into the degenerated 23-mm Lotus valve. Then, post-dilatation with a $20 \mathrm{~mm}$ balloon was carried out. The whole procedure was thoroughly prepared as shown in Figures $1 \mathrm{~A}$ and $\mathrm{B}$. The patient was able to walk on the evening after the procedure. Postprocedure transthoracic echocardiography showed a reduction of transaortic gradients to $16 / 8 \mathrm{~mm} \mathrm{Hg}$. After a 3-month follow-up the transaortic gradient stayed low and the patient's functional NYHA class improved to II.

\section{Discussion}

This case shows a unique set of catheterization therapies performed over two decades. The patient underwent ASA. Years later she developed severe aortic stenosis and had TAVI which degenerated early and required TAVI-in-TAVI. Furthermore, we present a favorable early outcome of TAVI-in-TAVI 23-mm Portico into a 23-mm Lotus valve. On one hand, the TAVI-in-TAVI procedure provides an important way to avoid high-risk surgery once the TAVI valve becomes degenerated [9]. On the other

Corresponding author:

MUDr. Eva Polaková, Charles University, Motol University Hospital, Prague, Czech Republic, e-mail: eva.polakova@fnmotol.cz

Received: 13.09.2021, accepted: 17.12.2021. 


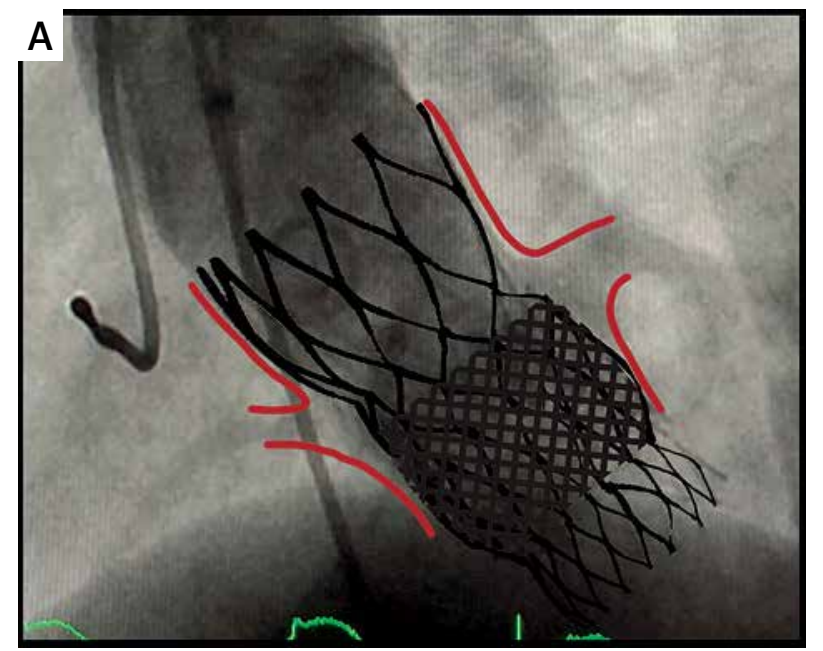

B

Figure 1. The stent frame of original Lotus valve was overlapping the coronary ostia. Therefore, the new valve had to be chosen very cautiously with regard to the index stent frame height. Since the height of 23-mm Lotus stent was $19 \mathrm{~mm}$ (A), the 23-mm Portico with nonflared anulus section height of $26 \mathrm{~mm}$ (B) was chosen

hand, it brings brand new challenges that we will need to cope with in the future. As patients with TAVI are younger and have a longer estimated lifespan, the need for safe coronary access becomes more pressing as the manifestation of coronary artery disease is more expectable. Also, occlusion of coronary ostia becomes a serious issue in redo TAVI procedures. The complexity of the procedure is a consequence of the anatomical and device related factors [6]. The preparation and implantation have to be done with great accuracy and precision. The stent frame of the original Lotus valve was overlapping the coronary ostia. The free flow to coronary arteries was ensured by side access outside the prosthetic stent owing to the high and wide sino-tubular junction. Therefore, the new valve had to be chosen very cautiously with regard to the index stent frame height. Since the height of the 23-mm Lotus stent was $19 \mathrm{~mm}$, the 23-mm Portico with nonflared anulus section height of $26 \mathrm{~mm}$ was chosen. Moreover, caution had to be taken regarding the depth of implantation, as the restriction of anterior mitral valve movement was feared.

\section{Conclusions}

This case is an evident example of an ongoing paradigm shift towards catheterization procedures in cardiovascular medicine. Moreover, to the best of our knowledge, we describe the first case of TAVI-in-TAVI 23-mm Portico into $23-\mathrm{mm}$ Lotus valve implantation. We have proven the clinical utility and technical feasibility of this procedure. We would like to stress the importance of exact and precise preprocedural preparation.

\section{Conflict of interest}

The authors declare no conflict of interest.

\section{References}

1. Sigwart U. Non-surgical myocardial reduction for hypertrophic obstructive cardiomyopathy. Lancet 1995; 346: 211-4.

2. Cribier A, Eltchaninoff $\mathrm{H}$, Bash A, et al. Percutaneous transcatheter implantation of an aortic valve prosthesis for calcific aortic stenosis: first human case description. Circulation 2002; 106: 3006-8.

3. Popma JJ, Deeb GM, Yakubov SJ, et al. Transcatheter aortic-valve replacement with a self-expanding valve in low-risk patients. N Engl J Med 2019; 380: 1706-15.

4. Mack MJ, Leon MB, Thourani VH, et al. Transcatheter aortic-valve replacement with a balloon-expandable valve in low-risk patients. N Engl J Med 2019; 380: 1695-705.

5. Makkar RR, Thourani VH, Mack MJ, et al. Five-year outcomes of transcatheter or surgical aortic-valve replacement. N Engl I Med 2020; 382: 799-809.

6. Buzzatti N, Romano V, De Backer O, et al. Coronary access after repeated transcatheter aortic valve implantation. JACC Cardiovascular Imaging 2019; 13: 508-15.

7. Dvir D, Leipsic J, Blanke P, et al. Coronary obstruction in transcatheter aortic valve-in-valve implantation preprocedural evaluation, device selection, protection, and treatment. Circ Cardiovasc Interv 2015; 8: e002079.

8. Willson AB, Rodès-Cabau J, Wood DA, et al. Transcatheter aortic valve replacement with the St. Jude Medical Portico valve firstin-human experience. JACC 2012; 60: 581-6.

9. Vrachatis DA, Vavuranakis M, Tsoukala S, et al. TAVI: valve in valve. a new field for structuralists? Literature review. Hell J Cardiol 2020; 61: 148-53. 